\title{
Using a zone model to incorporate the influence of geometry on polychromatic speckle contrast
}

\author{
Lioudmila Tchvialeva \\ Tim K. Lee, MEMBER SPIE \\ Igor Markhvida \\ David I. McLean \\ Harvey Lui \\ Haishan Zeng, MEMBER SPIE \\ University of British Columbia \\ Department of Dermatology and Skin Science \\ Photomedicine Institute \\ and \\ Vancouver Coastal Health Research Institute \\ and \\ British Columbia Cancer Research Centre \\ Cancer Imaging Department and Cancer \\ Control Research Program \\ 835 West 10th Avenue \\ Vancouver, BC, Canada, V5Z 4E8 \\ E-mail: tlee@bccrc.ca
}

\begin{abstract}
A simple physical zone model was developed to explain the formation of polychromatic speckle patterns within the Fresnel region. This model represents a reasonable compromise between complex theoretical formulation and simple estimations for practical needs, and allows the speckle contrast to be calculated as a function of geometric parameters for the optics and coherence length of the light source. The model was experimentally verified, and the results are consistent with our previous rigorous theoretical formulation. () 2008 Society of Photo-Optical Instrumentation Engineers. [DOI: 10.1117/1.2955797]
\end{abstract}

Subject terms: speckle contrast; low-coherence source; Fresnel diffraction; polychromatic speckle.

Paper 080033R received Jan. 15, 2008; revised manuscript received Apr. 28, 2008; accepted for publication Apr. 29, 2008; published online Jul. 14, 2008. This paper is a revision of a paper presented at the SPIE conference on Speckles, from Grains to Flowers, September 2006, Nimes, France. The paper presented there appears (unrefereed) in SPIE Proceedings Vol. 6341.

\section{Introduction}

Although the contrast of polychromatic speckle patterns has been used for numerous practical applications, ${ }^{1-13}$ there are still situations, such as Fresnel diffraction, where simple and practical analytical expressions have not yet been derived. To fill this gap we have developed a simple geometrical model utilizing a Fresnel-zone analogy for quantitative estimation of the contrast.

The speckle contrast is of great interest in various industrial ${ }^{1,2}$ and biomedical applications. ${ }^{3-10}$ By measuring its value as a function of either tissue thickness or the coherence length of the light source, the absorption and scattering coefficients of the diffusive media can be derived. ${ }^{4}$ Temporal averaging of contrasts for dynamic biospeckle has been used to monitor tissue conditions, ${ }^{5}$ visualize internal inhomogeneities, ${ }^{6-9}$ and evaluate blood flow velocity. ${ }^{10,11}$

From a metrological point of view, the speckle contrast contains useful information when it differs from unity. It is well known that for all interference phenomena contrast reduction occurs when a coherent addition of light waves starts to compete with an incoherent one. This effect takes place when the mean optical-path-length difference is of order smaller than the coherence length of the light source.

Variations of the ratio of optical-path-length differences to coherence lengths can have a number of causes. Some of them, such as surface roughness and low coherence of the illuminating light, have been studied. ${ }^{12}$ Most analytical solutions for these studies were obtained for the far-field diffraction zone. ${ }^{12-14}$ In another study a general formula for

0091-3286/2008/\$25.00 @ 2008 SPIE the intensity correlation function, based on the theory of linear systems, was considered, and analytical formulas were derived for the imaging geometry. ${ }^{15}$

In a previous paper ${ }^{16}$ we studied optical geometry as another factor that can significantly influence the contrast of polychromatic speckle within a Fresnel diffraction zone. In this article we extend this approach by developing a simple physical model of speckle formation that is an analogy of the Fresnel-zone technique.

This paper is organized as follows: after a short review (Sec. 2) on the background concept of forming polychromatic speckles as a sum of $N$ independent speckle patterns, ${ }^{12,17,18}$ we show how to construct zones geometrically in such a way that each zone creates an individual fully developed speckle (Sec. 3). The model is applied to contrast calculations, and some immediate practical applications are discussed. Next we prove that the model is consistent with our previous theoretical formulation ${ }^{16}$ (Sec. 4). Finally, experimental validation using different light sources-including a diode laser (DL) and a relatively new type of low-coherence light source, a superluminescence diode (SLD)—is presented (Sec. 5).

\section{Background}

The contrast $C$ of a speckle pattern ${ }^{12}$ is defined as

$C=\frac{\left(\left\langle I^{2}\right\rangle-\langle I\rangle^{2}\right)^{1 / 2}}{\langle I\rangle}$,

where $I$ is the light intensity and $\langle\cdots\rangle$ denotes ensemble averaging.

The simplest speckle, which is also known as fully developed or Gaussian speckle, is created by coherent monochromatic laser light. The contrast $C$ of this kind of speckle equals unity and does not depend on any physical param- 
eters. In other words, it does not carry information about the scattering medium or optical geometry. However, analyzing speckle patterns where the contrast differs from unity allows us to get useful information about the sample under investigation.

Goodman $^{12}$ shows that the contrast $C$ of $N$ independent and fully developed speckle patterns each having equal mean intensity $\left\langle I_{n}\right\rangle=I_{0}$ can be expressed as

$C=\frac{1}{\sqrt{N}}$

The main advantage of this formula is its generality. To derive it, only universal statistical concepts were used, and it does not involve any physical parameters. This generic approach allows for the contrast to be determined from complex speckle phenomena that would not otherwise have had any conventional analytical description. For example, one can take into consideration individual contrast reduction factors as well as their combined effects. On the other hand, determining $N$ may be a challenge for practical applications.

The known sources of speckle contrast reduction are coherence (angular and wavelength diversity), polarization diversity, temporal and spatial averaging, and others. Some of these factors lead to the generation of independent speckle patterns. Examples of determining $N$ [Eq. (2)] for contrast reduction due to increased surface roughness can be found in Refs. 13 and 14 as side remarks on contrast calculation. In Refs. 17 and $18 \mathrm{~N}$ is calculated for polychromatic imageplane speckle.

Below, we have reformulated the results of Refs. 13 and 14 to emphasize the concept of exploiting the same generic approach of polychromatic speckle formation despite the fact that the optical situations are completely different: Specifically, the preceding studies ${ }^{13,14}$ investigated speckle in the Fraunhofer diffraction zone, whereas we are primarily interested in light properties outside of the Fraunhofer zone.

The general solution for $C$ in Refs. 13 and 14 is reduced to

$C^{2}=\frac{1}{\left[1+\left(2 \sigma_{\Delta} \sigma_{k}\right)^{2}\right]^{1 / 2}}$,

where $\sigma_{k}$ is the effective width of the Gaussian spectral profile of the illumination source, and $\sigma_{\Delta}$ is the root mean square (rms) of the optical-path-length deviation. Equation (3) is valid for either reflectance and transmittance schemes; for reflection $\sigma_{\Delta}=2 \sigma_{h}$ and $\sigma_{h}$ is the rms height deviation of the diffuser, and for transmittance $\sigma_{\Delta}=(n$ -1) $\sigma_{h}$ with $n$ the medium's refractive index. When $\sigma_{k}$ has a Gaussian spectral profile, it can be expressed in terms of the temporal coherence length $L_{c}$ as $\sigma_{k}=\sqrt{ } / L_{c}$. In the case of $2 \sigma_{\Delta} \gg 1 / \sigma_{k}$, comparing Eqs. (2) and (3), we can rewrite $N$ as

$N \sim \Delta / L_{c}$,

where $\Delta=2 \sigma_{\Delta}$ representing the total mean optical path difference.

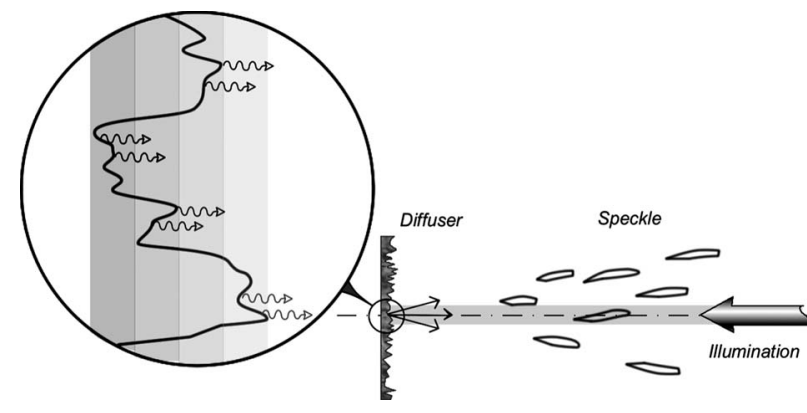

Fig. 1 Formation of polychromatic speckle by light reflected from rough surface.

Figure 1 helps us interpret the physical meaning of Eq. (4). A light source illuminates a rough surface from the left side. Scattered light creates a speckle pattern in the Fraunhofer field. Consider that the rough surface can be split vertically into layers of thickness $L_{c} / 2$. Different layers are depicted by different gray tones in the inset of Fig. 1. All elementary waves scattered from a single layer are considered mutually coherent. They create a fully developed speckle pattern with unit contrast. Similarly, the elementary waves reflected from the next layer are mutually coherent and generate the second speckle pattern. To simplify the analysis, we consider that the first and second speckle patterns are naturally incoherent and do not interfere with each other. Analogously, we can form the third and additional subsequent speckle patterns up to $N$ $=\beta(\Delta / 2) /\left(L_{c} / 2\right)$, where $\beta$ depends on the spectral profile and is of the order of unity. Overlaying these $N$ speckle patterns results in the final pattern with a reduced contrast.

In summary, the appropriate choice of coherent layers allows us to model the formation of a speckle pattern in a simple manner and to calculate its contrast. The ratio $\Delta / L_{c}$ is a key element in the formulation, and we follow the same rationale for the following sections.

\section{Zone Model}

The coherence length is closely related to the mutual coherence function. Like wavelength, amplitude, phase, and polarization, the mutual coherence function is one of a wave's basic characteristics, which carries information about the wave and about the objects or processes that the wave interacts with during its propagation. Recently a class of new measuring techniques utilizing mutual coherence functions has been established. These techniques cover areas such as low-coherence holography, low-coherence speckle, low-coherence tomography, and others. ${ }^{19}$ Like amplitude, the mutual coherence function obeys wave equations. This fact gives us an opportunity to develop an analogy to interference measurements, where the coherence length behaves similarly to the effective wavelength. Utilizing that analogy, we thus propose a multizone construction for polychromatic speckling.

The polychromatic speckle contrast degrades not only because of roughness but also because of geometry factors. When an illuminated area is large enough and the diffusersource distance or the receiver-source distance is finite, the optical path length of a light ray passing through the peripheral zone can differ significantly from the optical path 


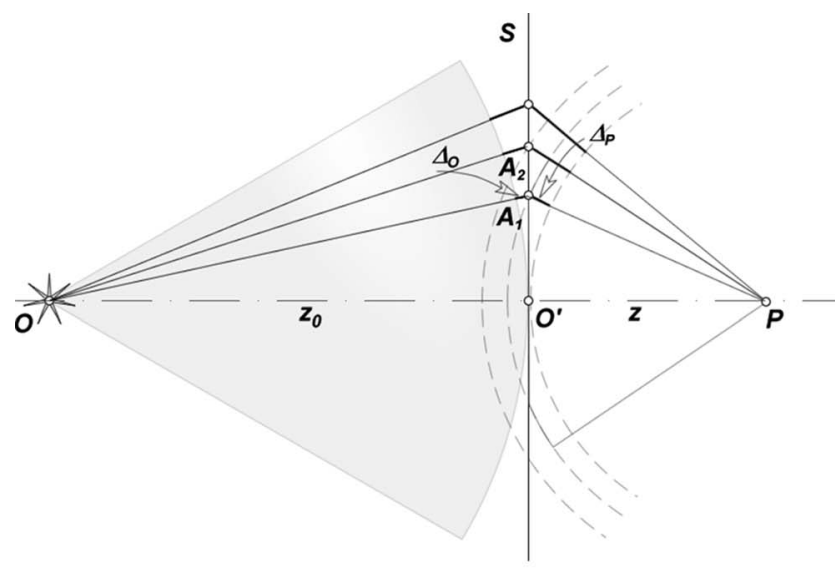

(a)

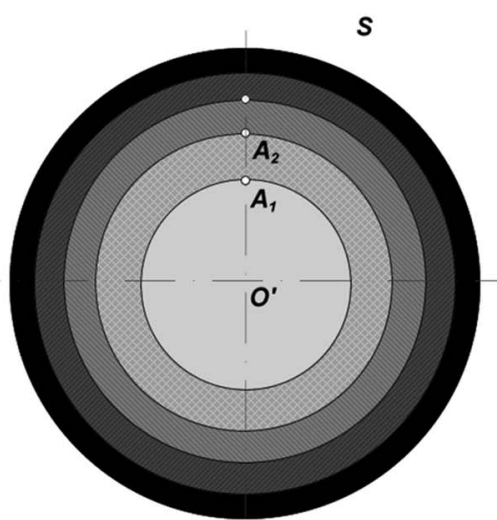

(b)

Fig. 2 Passage of light through the central and peripheral parts of an illuminated surface $S$ : (a) side view along the optical axis; (b) front view of the illuminated surface $S$.

length through the central part of the surface. If the opticalpath-length difference is greater than the coherence length, rays will not interfere with each other.

Let us calculate the contrast due to the mentioned geometrical consideration as a sum of $N_{\text {geom }}$ fully developed patterns where each pair of patterns is mutually incoherent. To do that we introduce coherent zones: each coherent zone creates one speckle pattern. A geometrical construction is shown in Fig 2. Coherent zones can be applied to both transmission and reflection geometry; however, only the transmission geometry is shown in Fig. 2, to better illustrate the optical pathways. We suppose that a light ray emitted from the point $O$ (for example, at the end of a singlemode fiber) comes to the point $P$ through a thin diffuser located in the plane $S$. We use a technique similar to the Fresnel zone construction. ${ }^{20}$ First, we draw a sphere centered at the point $O$ with radius $z_{0}$. Then we draw another sphere centered at $P$ in the opposite direction with radius $z$. Note that $z_{0}+z$ is the optical path of the ray that passes through the central point $O^{\prime}$ on the surface $S$. Let us choose a point $A_{1}$ that provides the ray route $O A_{1} P$ with the optical path length $z_{0}+\Delta_{0}+\Delta_{P}+z$. We choose $A_{1}$ in such a way that the optical-path-length difference between $O P$ and $O A_{1} P$ is of the order of the light-source coherence length, i.e., $\Delta_{0}$ $+\Delta_{P}=L_{c}$. We define the coherence length ${ }^{21}$ as $L_{c}$ $=c \int_{-\infty}^{\infty}|\gamma(\tau)|^{2} \mathrm{~d} \tau$, where $\gamma(\tau)$ is the coherence function and $c$ is the light speed. The new sphere with radius $P A_{1}$ crosses the diffuser $S$, creating a circle with the radius $O^{\prime} A_{1}=R_{1}$. Simplifying somewhat, we assume that the rays that pass through different points within this zone and reach the observer $P$ are coherent. The actual optical path of each ray includes a stochastic component related to the roughness of $S$, but this will not influence our construction as long as the roughness height remains small compared to the coherence length. Interference of all these rays creates the first fully developed speckle pattern.

Continuing our construction, let us find another point $A_{2}$ that limits the second zone $\left(O^{\prime} A_{2}=R_{2}\right)$, where $\Delta_{02}+\Delta_{P 2}$ $=2 L_{c}$. Because of the circular symmetry, any pair of rays passing through the second zone (which is a ring) will have a path-length difference that is less than the coherence length. All these rays create the second fully developed speckle pattern.

Other zones are constructed in the same fashion. Each zone creates an independent speckle pattern and contributes to the overall resultant pattern, wherein the contrast is reduced. Simple geometrical calculations show that the radius of zone $N$ is approximately

$R_{N}^{2}=\frac{2 L_{c} z_{0} z}{z_{0}+z} N$

The total number of coherent zones due to the geometry influence, $N_{\text {geom }}$, is limited by the spot size $q$ of the illuminated area on the surface and can be calculated from the equation $R_{N}=q$ :

$N_{\text {geom }}=\frac{q^{2}\left(z_{0}+z\right)}{2 L_{c} z_{0} z}=\frac{\Delta_{\text {geom }}}{L_{c}}$.

Equation (6) is valid for the reflectance as well. It is easy to show that the area $S_{n}$ of each flat ring zone is approximately the same: $S_{1} \approx S_{2} \approx \cdots \approx S_{N}$ (to prove that, we refer the reader to the Fresnel zone model $\left.{ }^{20}\right)$. Therefore, if the illumination is uniform, each zone transmits (or reflects) equal amount of light, and the average intensity of each speckle pattern is the same. Our goal is to determine the contrast $C_{\text {geom }}$ due to geometry-based parameters. Combining Eqs. (6) and (2), and introducing $z_{\mathrm{eff}}=z z_{0} /\left(z+z_{0}\right)$, we obtain

$C_{\mathrm{geom}}^{2}=\frac{2 z_{\mathrm{eff}} L_{c}}{q^{2}}$.

Eq. (7) shows that the contrast is reduced when $q$ increases or when the coherence length and the effective distance $z_{\text {eff }}=z z_{0} /\left(z+z_{0}\right)$ decrease. The range of experimental parameters in Eq. (6) obeys the natural limitation $N_{\text {geom }}$ $>1$ (the number of summed patterns must be greater than one), which is the same as for Eq. (2). 
Certain features concerning the generalization and application of this model can be observed:

- The model can be applied to other geometries. The simplest example is a rectangular slit. The construction of the zones should be analogous to the construction of Fresnel zones for the required geometry.

- Fractional $N$ can be considered with appropriate changes in Eq. (2). See Ref. 12 for summing $N$ speckle patterns with unequal average intensities.

Speckle contrast reduction can be easily observed when modern low-coherence light sources are applied with a large illumination spot and a small target distance. In practical terms this can be implemented with optical fiber illumination. Another obvious application of Eqs. (6) and (7) is that the effective coherence length of a light source can be obtained without spectral devices, but rather through realtime speckle contrast measurements.

\section{Theory}

Our proposed model is consistent with a rigorous application of diffraction theory. If we illuminate a diffuser with a polychromatic light source characterized by half-width $\Delta \lambda$, each wavelength produces a shifted speckle pattern and the final interference pattern becomes blurry. The intensity $I$ of the polychromatic speckles is the sum of the monochromatic speckle pattern intensities,

$I(x)=\int_{0}^{\infty} F(k) I(\mathbf{x}, k) \mathrm{d} k$,

where $k$ is the wave number, $F(k)$ is the spectral line profile of the illumination light, and $\mathbf{x}$ is a vector in the observation plane $P$. Equation (8) implies that the exposure time is much greater than the coherence time. In other words, speckle patterns created by individual wavelengths are incoherent, and we sum them on an intensity basis. The general expression for the contrast in Eq. (1) taking into account of Eq. (8) can be rewritten as

$C^{2}=\frac{\int_{0}^{\infty} \int_{0}^{\infty} F\left(k_{1}\right) F\left(k_{2}\right)\left[\left\langle I\left(\mathbf{x}, k_{1}\right) I\left(\mathbf{x}, k_{2}\right)\right\rangle-\left\langle I\left(\mathbf{x}, k_{1}\right) I\left(\mathbf{x}, k_{2}\right)\right\rangle\right] \mathrm{d} k_{1} \mathrm{~d} k_{2}}{\left|\int_{0}^{\infty} F(k)\langle I(\mathbf{x}, k)\rangle \mathrm{d} k\right|^{2}}$.

To calculate Eq. (9) we applied the Fresnel approach of diffraction theory for free-space geometry (Fig. 2). ${ }^{16}$ The correlation of intensity fluctuations in this case can be written as

$$
\begin{aligned}
& \left\langle I\left(\mathbf{x}, k_{1}\right) I\left(\mathbf{x}, k_{2}\right)\right\rangle-\left\langle I\left(\mathbf{x}, k_{1}\right)\right\rangle\left\langle I\left(\mathbf{x}, k_{2}\right)\right\rangle \\
& \quad=\left|\int \exp \left[i\left(k_{1}-k_{2}\right) R\right]\left\langle\exp \left[i\left(k_{1}-k_{2}\right) \Delta_{h}(\zeta)\right]\right\rangle \mathrm{d} S\right|^{2},
\end{aligned}
$$

where $R$ is the total optical path of a wave from $O$ to $P$ along ray $O A P$. We assumed the following: The spectral line has a Gaussian profile, the roughness height of the diffuser has a Gaussian distribution, the roughness is larger than the wavelength but smaller than the coherence length, and the light intensity is evenly distributed across the diffuser surface. Calculations ${ }^{16}$ yield the following results:

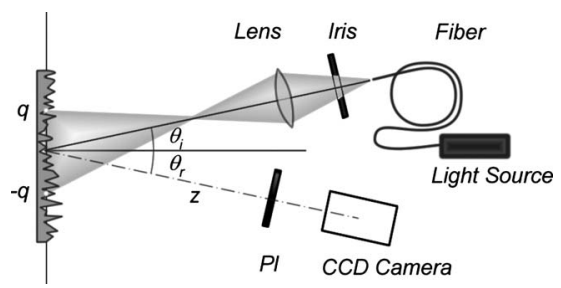

Fig. 3 The experimental setup.

$C^{2}=0.664 \frac{2 z_{\mathrm{eff}} \lambda^{2}}{q^{2} \Delta \lambda}$

where $q$ is the size of the illuminated spot, and $z_{\mathrm{eff}}$ $=z z_{0} /\left(z+z_{0}\right)$. For a spectral line with Gaussian profile, $L_{c}$ $=0.664 \lambda^{2} / \Delta \lambda,{ }^{21}$ and Eq. (11) is equivalent to Eq. (7).

Some important simplifications were made in order to obtain Eq. (11). We considered the case when the mean optical path difference is greater than the coherence length and the surface roughness. Also, to obtain an analytical solution we extended the integral limits to infinity, implying the condition $q^{4} /\left(z_{\mathrm{eff}} L_{c}\right)^{2} \gg 1$. This condition dictates the parameter ranges of Eq. (11) and leads to $C<1$. Therefore, this formula describes an experimental situation with a wide illuminating beam, a low-coherence source, and a short source-detector distance.

The performed analysis shows that despite a number of simplifications, the zone model agrees well with the results of a rigorous application of theory.

\section{Experiment}

An experimental validation of our model involves finding the contrast from the calculated number $N_{\text {geom }}$ of coherent zones and comparing the experimental values of $C_{\text {geom }}$ with the theoretical expectation as expressed in Eq. (2). The value of $N_{\text {geom }}$ is determined by Eq. (6) according to variations in the geometric parameters $z_{\text {eff }}$, and $q$.

The free-space geometry setup is shown in Fig. 3. Divergent waves illuminate a rough sample. Two lowcoherence light sources were tested: a superluminescent diode (FSLD-3P-680, RPMC Inc., USA, $\lambda=683.6 \mathrm{~nm}$ with a half-width spectrum line $\lambda=9.5 \mathrm{~nm}$ and close to Gaussian spectral profile) with coherence length calculated as $L_{c}$ $=33.2 \mu \mathrm{m}$; and a laser diode (57PNL053/P4/SP, Melles Griot, USA, $\lambda=656 \mathrm{~nm}$, multipeak spectral profile) with coherence length measured as $L_{c}=278 \mathrm{~mm}$. The lens and iris were used to adjust the size of the illuminated spot $(q)$ and $z_{\text {eff }}$.

A CCD camera without a lens recorded speckle patterns. We captured the spatial homogeneous portion of a speckle pattern. Therefore the ensemble averaging in Eq. (1) could be replaced by spatial averaging of all CCD camera pixels. The 8 -bit CCD sensor had $512 \times 486$ pixels, and the pixel size was made 5 times smaller than the average speckle size to avoid spatial averaging of the speckle contrast. The camera circuit allowed regulation of the dark current and gain, which were adjusted to supply a linear measurement mode.

The setup was designed mostly to remove contrast reduction factors except for the geometry parameters. In particular, the dark-current pedestal was subtracted from the 


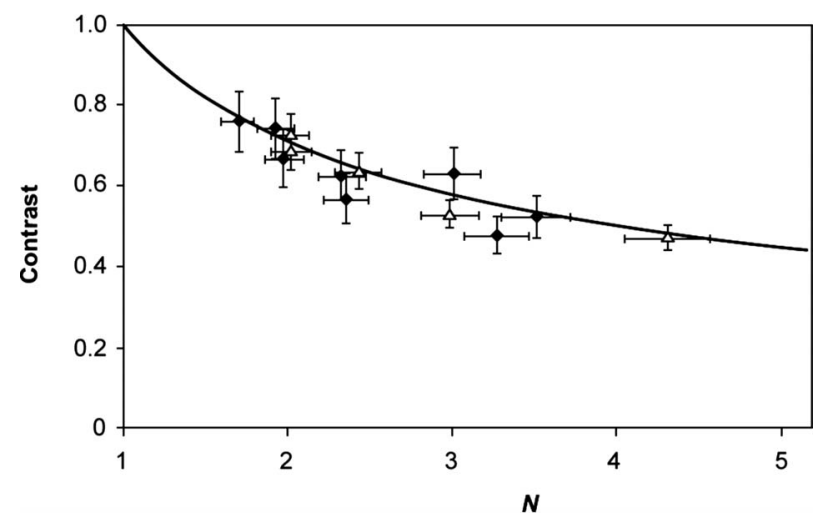

Fig. 4 The dependence of the speckle contrasts on the number of coherent zones. Triangles are the DL measurements, diamonds are the SLD data, and the solid line is the theoretical expectation.

speckle images. Any contrast reduction due to light depolarization was controlled by an output polarizer Pl. The angles of incidence $\left(\theta_{i}\right)$ and reflection $\left(\theta_{r}\right)$ were almost identical. We used metal roughness standards with profound roughnesses of 6 and $3.17 \mu \mathrm{m}$ to reduce the specular component of the reflected light, which lowers the contrast. The roughness is still considerably less than the effective coherence lengths for both the DL and SLD light sources. Therefore, we can ignore the surface roughness effect in evaluating contrast changes.

The measured contrast versus the number of coherent zones [as calculated by Eq. (6)] is plotted in Fig. 4. For both light sources the experimental points are consistent with the theoretical curve (solid line) $C=1 / \sqrt{N}$, which validates Eqs. (6) and (7). Note that the SLD contrast values display a slight downtrend. This degradation may be caused by the surface roughness due to the shorter $L_{c}$ of the SLD than the LD. More accurate theoretical calculation and modeling of compounding contrast, taking into account the combination of geometry, roughness, and other possible contrast reduction factors, is our next goal.

\section{Conclusion}

We have developed a zone model to explain quantitatively the polychromatic speckle contrast reduction when the geometry involves path-length differences large compared to the surface roughness of a diffuser. The model is analogous with the Fresnel-zone construction, with wavelength replaced by coherence length. The model is consistent with our theoretical results ${ }^{16}$ and has been validated experimentally.

Investigating the sources of contrast reduction is especially important for modern low-coherence light sources with short temporal coherence. The ideas and the formulas discussed may find immediate applications in measuring temporal coherence lengths without spectral devices, measuring geometrical parameters, and assisting experimental design to eliminate or suppress speckle. The proposed model could also be useful for separating different contrast reduction factors in situations involving complex contrast formation.

\section{Acknowledgments}

This work was supported in part by a Canadian Dermatology Foundation grant, a Proof-of-Principle Grant from the Canadian Institutes of Health Research (PPP-82309), and a Discovery Grant from the Natural Sciences and Engineering Research Council of Canada (288194-06).

\section{References}

1. K. Leonhardt and H. J. Tiziani, "Removing ambiguities in surface roughness measurement," Opt. Acta 29(4), 493-499 (1982).

2. C. Hun, J. Caussignac, and M. Brynooghe, "Speckle techniques for pavement surface analysis," Proc. SPIE 4933, 261-266 (2003).

3. F. Ciang, "Evolution of white light speckle method and its application to micro/nanotechnology and heart mechanics," Opt. Eng. 42(5), 1288-1292 (2003).

4. J. McKinney, M. Webster, K. Webb, and A. Weiner, "Characterization and imaging in optically scattering media by use of laser speckle and a variant-coherence source," Opt. Lett. 25, 4-6 (2000).

5. D. Zimnyakov, D. Agafonov, A. Sviridov, A. Omel'chenko, L. Kuznetsova, and V. Bagratashivili, "Speckle-contrast monitoring of tissue thermal modification," Appl. Opt. 41, 5989-5996 (2002).

6. P. Yu, M. Mustata, J. Turek, P. French, M. Melloch, and D. Nolte, "Holographic optical coherence imaging of tumor spheroids," Appl. Phys. Lett. 83, 575-577 (2003).

7. G. Tearney and B. Bouma, "Atherosclerotic plaque characterization by spatial and temporal speckle pattern analysis," Opt. Lett. 27, 533535 (2002).

8. J. Li, G. Ku, and L. Wang, "Ultrasound-modulated optical tomography of biological tissue by use of contrast of laser speckle," Appl. Opt. 41, 6030-6035 (2002).

9. R. Nothdurft and G. Yao, "Imaging obscured subsurface inhomogeneity using laser speckle," Opt. Express 13, 10034-10039 (2005).

10. H. Cheng, Q. Luo, Z. Wang, H. Gong, S. Chen, W. Liang, and S. Zeng, "Efficient characterization of regional mesenteric blood flow by use of laser imaging," Appl. Opt. 42, 5759-5763 (2003).

11. L. V. Tanin, S. C. Dick, S. A. Alexandrov, I. V. Markhvida, and L. A. Vasilevskaya, "Laser specklometer for determining the biomechanica parameters of skeletal muscles and the microhaemodynamics of human skin," Proc. SPIE 2769, 94-100 (1996).

12. J. W. Goodman, Speckle Phenomena in Optics: Theory and Applications, Roberts \& Company (2006).

13. H. M. Pendersen, "On the contrast of polychromatic speckle pattern and its dependence on surface roughness," Opt. Acta 22, 15-24 (1975).

14. G. Parry, "Speckle pattern in partially coherent light," in Laser Speckle and Related Phenomena, Springer-Verlag, Berlin, New York (1984).

15. N. George and A. Jain, "Space and wavelength dependence of speckle intensity," Appl. Phys. 4, 201-212 (1974).

16. I. Markhvida, L. Tchvialeva, T. K. Lee, and H. Zeng, "The influence of geometry on polychromatic speckle contrast," J. Opt. Soc. Am. A 24, 93-97 (2007).

17. J. Huntley, "Simple model for image-plane polychromatic speckle contrast," Appl. Opt. 38(11), 2212-2215 (1999).

18. N. G. Vlasov, E. G. Semenov, and M. E. Sokolova, "Imaging based on coherent properties of probe emission," in XVII National Holography School, Leningrad Institute of Nuclear Physics (1986).

19. C. Rodrigues and J. Pinto, "Contrast of polychromatic speckle patterns and its dependence to surface height distribution," Opt. Eng. 42, 1699-1703 (2003)

20. M. Born and E. Wolf, Principles of Optics, 6th ed., Pergamon Press (1980).

21. J. W. Goodman, Statistical Optics, John Wiley \& Sons (1985).

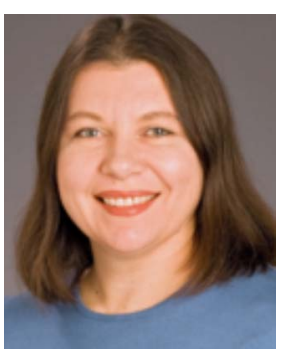

Lioudmila Tchvialeva (Chvyaleva) received her $\mathrm{PhD}$ in physics and mathematics from the Institute of Physics, Belarusian National Academy in 1990. Her research interests are optical diagnostics and biomedical optics. Currently she is doing research in the Photomedicine Institute and lecturing in UBC, Vancouver, Canada. 


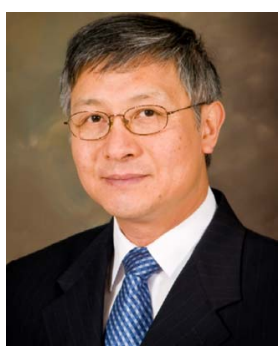

Tim K. Lee received his $\mathrm{PhD}$ in computer science in 2001 . He is currently a senior scientist at the Cancer Control Research Program, BC Cancer Agency; an assistant professor in the Department of Dermatology and Skin Science, University of British Columbia; and an adjunct professor in the School of Computing Science, Simon Fraser University. His research interests include computer-aided diagnosis and prevention of skin cancer. He is a member of SPIE.

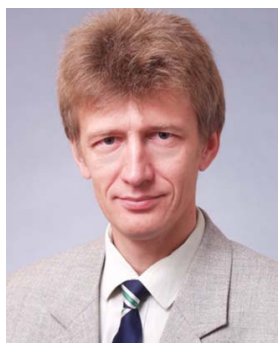

Igor Markhvida received his $\mathrm{PhD}$ in physics and mathematics from Belarusian State University, Minsk, in 1989. His research interests include coherent optics and speckle, microscopy, and image analysis. Currently he develops low-coherence measuring systems in association with the Photomedicine Institute, UBC, Vancouver, Canada.

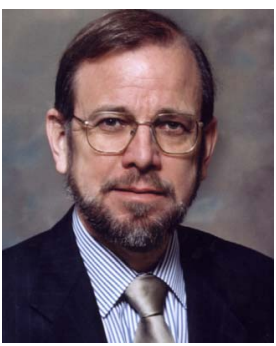

David I. McLean is a professor in the Department of Dermatology and Skin Science at the University of British Columbia, Vancouver, and is the head of Cancer Prevention at the British Columbia Cancer Agency. $\mathrm{He}$ has a special interest in the machine diagnosis of skin cancer.

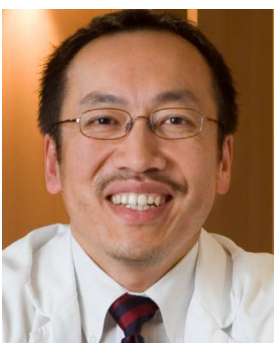

Harvey Lui is a professor and the head of the Department of Dermatology and Skin Science at the University of British Columbia. Dr. Lui was awarded the Killam Prize for his teaching and for establishing a fellowship training program in photomedicine,. He has been the principal investigator in more than 35 research projects, and has published more than 70 journal articles and more than 100 abstracts. Dr. Lui is the director of the CIHR Skin Research Training Centre and the VGH Photomedicine Institute. His clinical and research expertise includes lasers, photomedicine, psoriasis, vitiligo, and dermatological education.

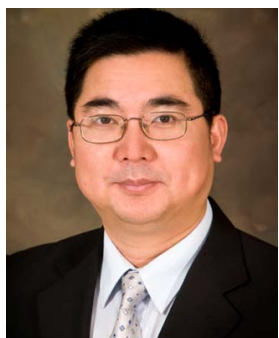

Haishan Zeng received a BSc degree in electronic physics from Peking University, Beijing, China, in 1983, a MASc degree in electronic physics and devices from the Institute of Electronics, Chinese Academy of Sciences, Beijing, China, in 1986, and a $\mathrm{PhD}$ degree in biophysics from the University of British Columbia, Vancouver, Canada, in 1993. He is now a senior scientist in the Cancer Imaging Department of the British Columbia Cancer Agency and an associate professor of medicine and physics at the University of British Columbia, Vancouver, Canada. For almost 20 years, he has been focused on developing various optical imaging and spectroscopy techniques for early cancer detection and for photodynamic therapy dosimetry. He has published 48 referred journal papers and 3 book chapters and obtained 18 patents related to optical diagnosis and therapy. He is a member of SPIE. 